\title{
REHABILITACIÓN AUDITORIO Facultad de Arquitectura y Urbanismo Universidad de Chile
}

\section{CONCURSO /}

Primer Premio

NOMBRE DE LA OBRA /

Rehabilitación Auditorio

Facultad de Arquitectura y Urbanismo

Universidad de Chile

LOCALIZACIÓN /

Santiago de Chile

ARQUITECTOS /

Rodrigo Aguilar P.

Malena Kaztman R.

Miguel Casassus R.

\section{COLABORADORES /}

Sebastián Laclabere A.

Claudia Oliva S.

ASESORÍA ACÚSTICA /

Leonardo Parma R.

UNIDAD TÉCNICA /

Centro de Proyectos Externos FAU

AÑO PROYECTO /

2011-2012

AÑO CONSTRUCCIÓN /

2012

SUPERFICIE PROYECTADA /

$220,00 \mathrm{~m}^{2}$

MATERIALIDAD /

Paneles de madera de terciado y pavimentos de porcelanato negro

FOTOGRAFÍAS /

Ricardo Martínez R. 
1. "Cazadores General Baquedano» y pisadas equinas. Patio actual de la Facultad de Arquitectura y Urbanismo de la Universidad de Chile.

2. Puerta de acceso al Auditorium.

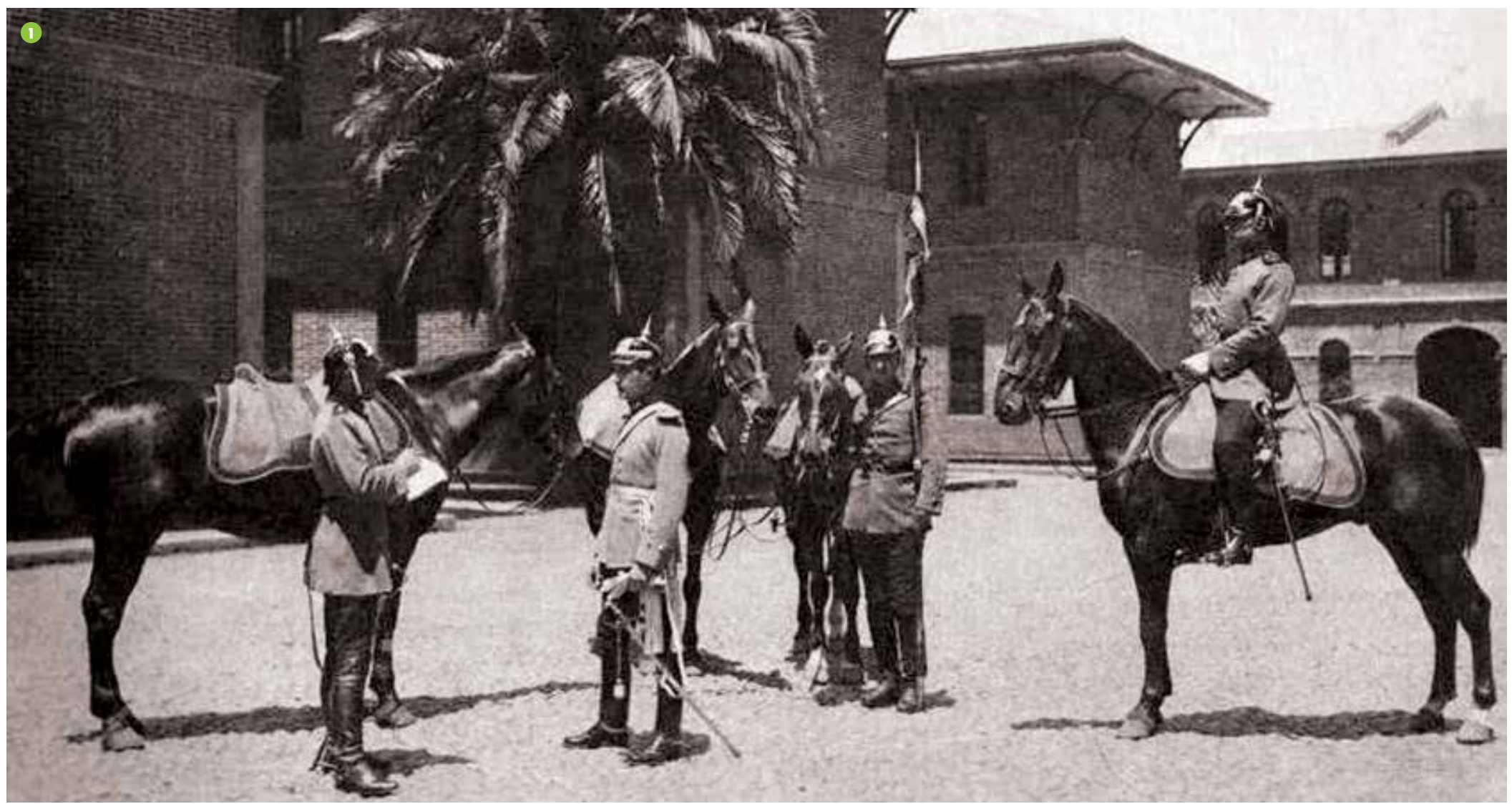

a propuesta para la rehabiltación del Auditorio - de la Facultad de Arquitectura y Urbanismo de la Universidad de Chile, se enmarca en el contexto del Proceso de Reestructuración de dicha unidad académica y la necesidad de adecuar y renovar sus espacios físicos en consideración a la nueva organización desplegada.

En relación a lo anteriormente expuesto, se intenta responder a la pregunta sobre cómo reflejar esta renovación en un ámbito de austeridad y simpleza, proveyendo de la infraestructura necesaria para un uso que se proyecta intensivo, estimulando el encuentro entre académicos y estudiantes, y mejorando además las condiciones acústicas y de visibilidad existentes.

Desde otra perspectiva, el proyecto se plantea como el pretexto para indagar sobre la forma de rescate de algunas preexistencias del edificio donde se inserta la propuesta. En efecto, los antiguos bloques de albañilería simple que desde el año 1976 ocupa la Facultad de Arquitectura y Urbanismo albergaron entre 1893 y 1933 el Cuartel del Regimiento de Caballería 2 «Cazadores» del Ejército de Chile, siendo el inmueble posteriormente habilitado para el Mercado Juan Antonio Ríos. Como vestigio de un inmueble con una data de más de un siglo, inserto en el corazón de lo que hoy se conoce como remodelación San Borja, el espacio de la Facultad se hace acreedor de un valor histórico y patrimonial considerable.

Tomando en cuenta estas premisas, la respuesta arquitectónica basa su ejecución en dos operaciones proyectuales esenciales: la primera de ellas intenta limpiar los estucos y las capas de revestimientos aplicados en el transcurso de tiempo al espacio interior, con la intención de restituir la construcción de albañilería simple de ladrillo con la cual había sido proyectado originalmente el bloque, dotando al lugar de una cierta sinceridad material que permite la lectura de una caja elemental para configurar el espacio principal del auditorio.

La segunda operación plantea la incorporación de una cinta continua y lineal de paneles de madera, que mediante una serie de pliegues, responde con su geometría a las necesidades de difusión del sonido, es capaz de ocultar los equipos técnicos necesarios y a la vez, permite configurar un plano inclinado que mejora la visibilidad hacia el lugar del escenario. La piel de madera que recorre suelo, muros frontales y cielo, proporciona una sensación global que unifica la comprensión del espacio, puesta en relieve a través de un ejercicio de flotación y se erige finalmente como un contrapunto entre la intervención nueva y los muros desnudos originales del edificio. 


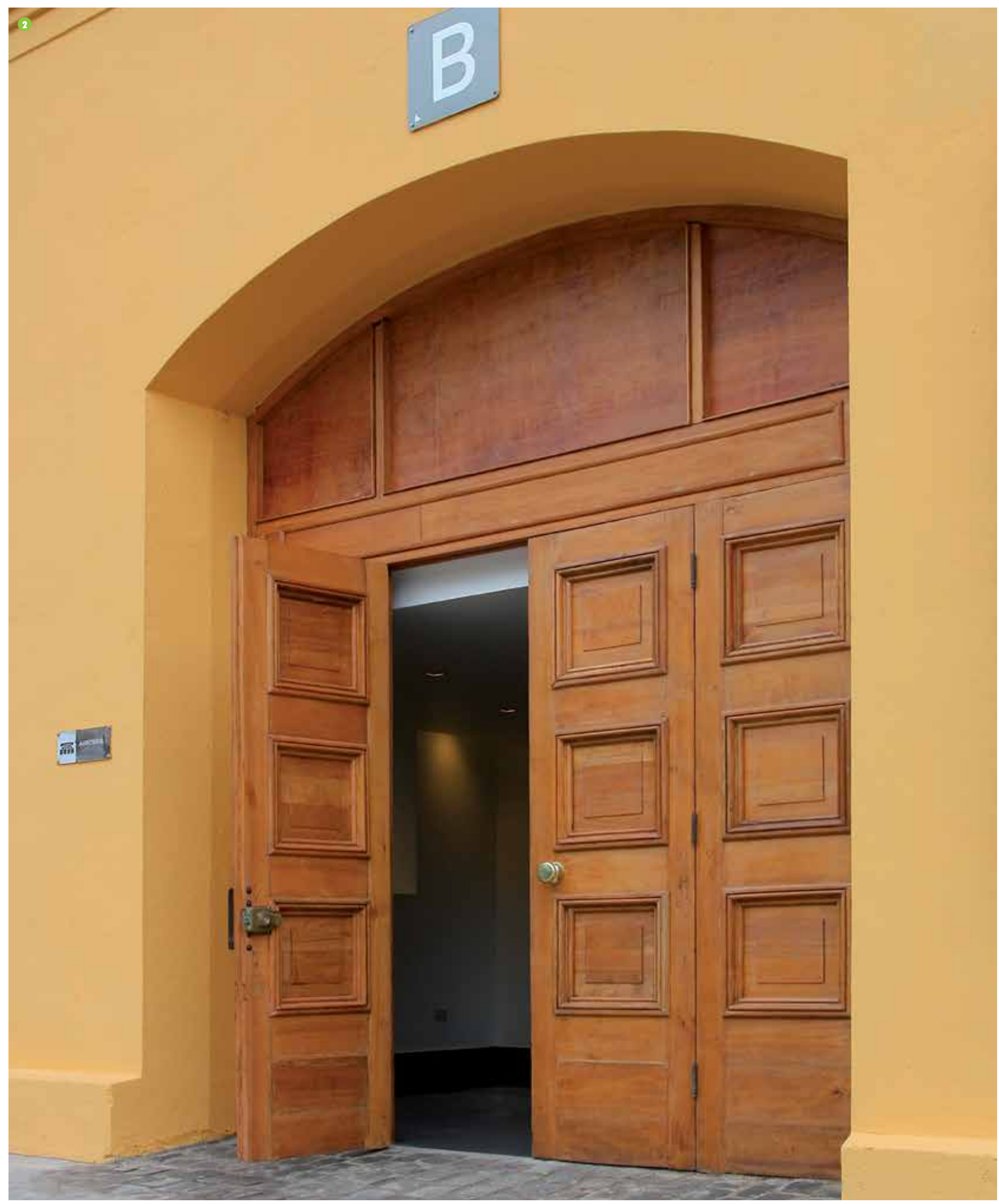




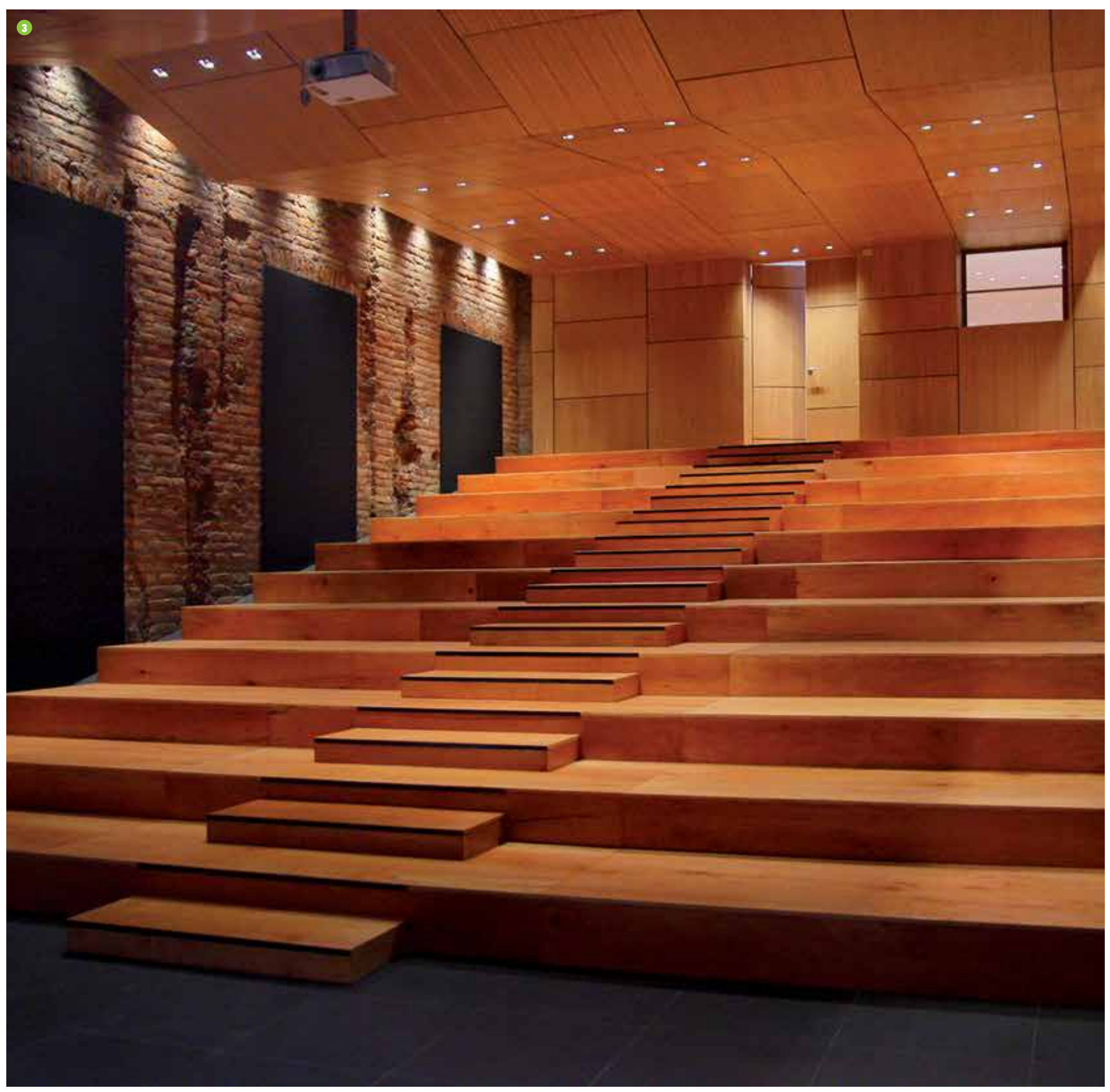


4. Vista hacia el escenario del Auditorium.

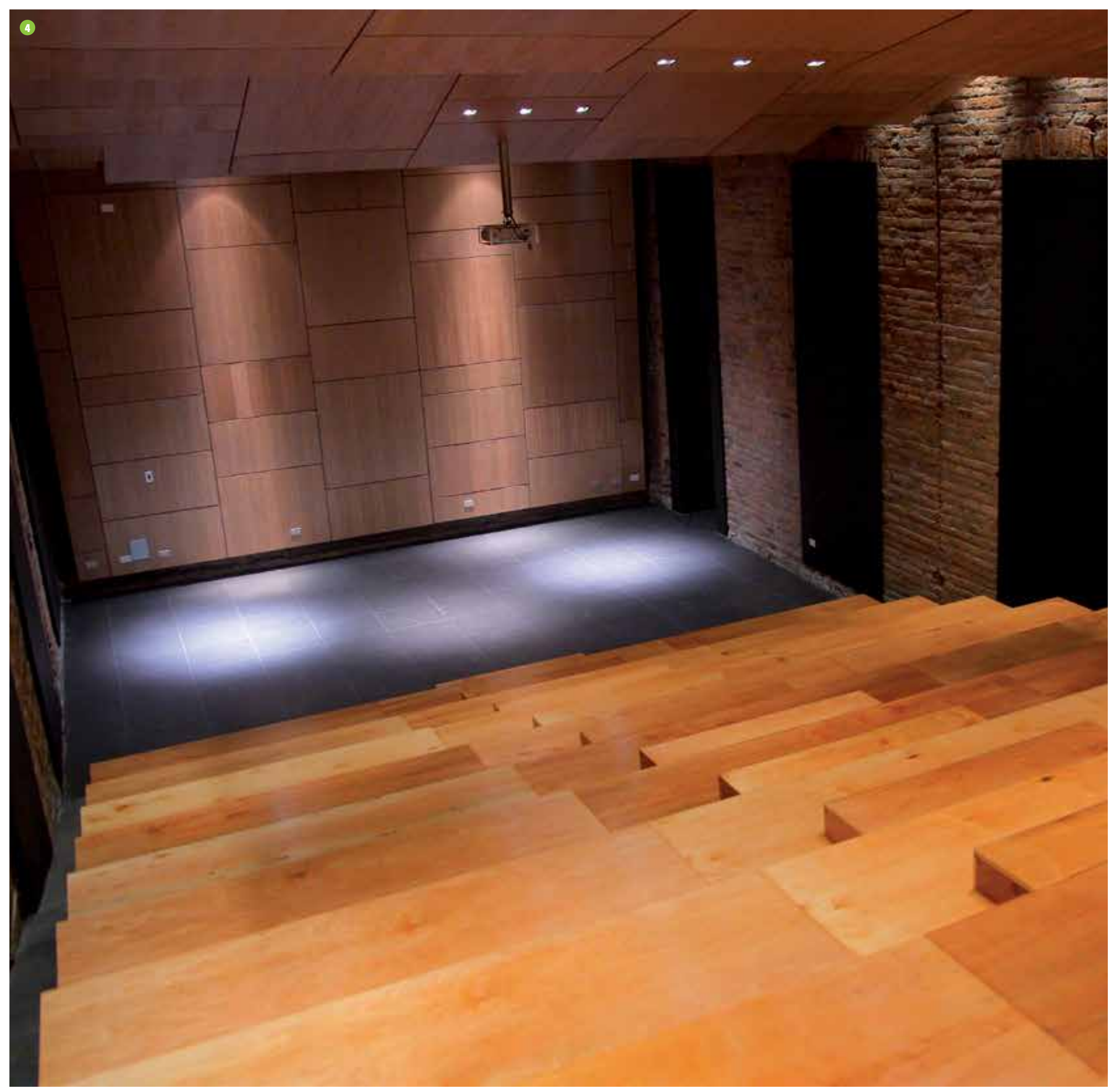




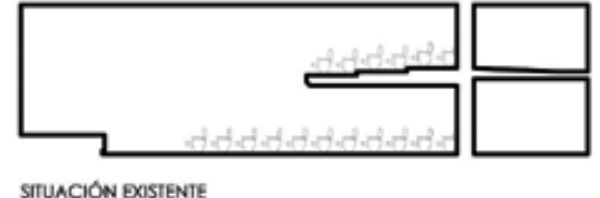

STIUACION ENSTENTE

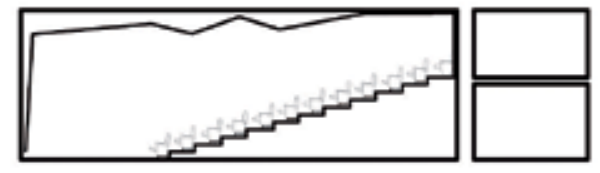

SIUACIÓN PROYECTADA

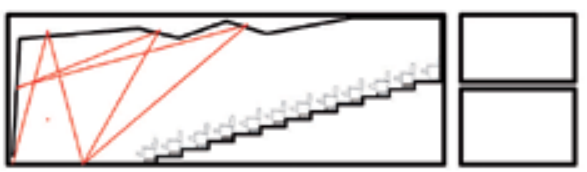

IUUMINACIÓN

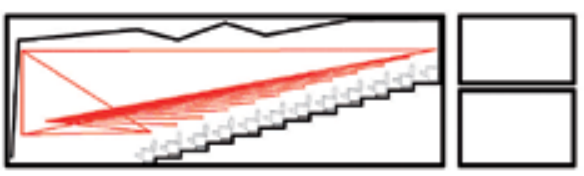

ACONDICIONAMIENTO VISUAL

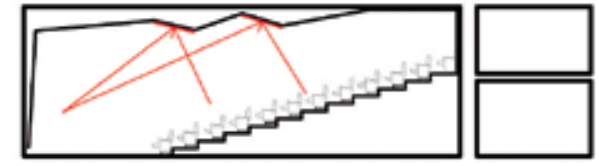

CONFORT ACÚSTICO

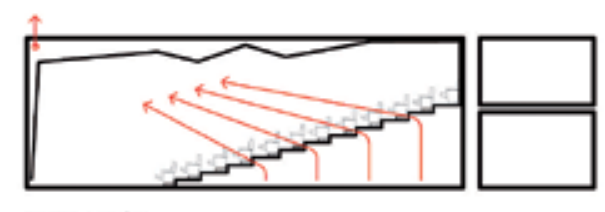

VENTILCIÓN

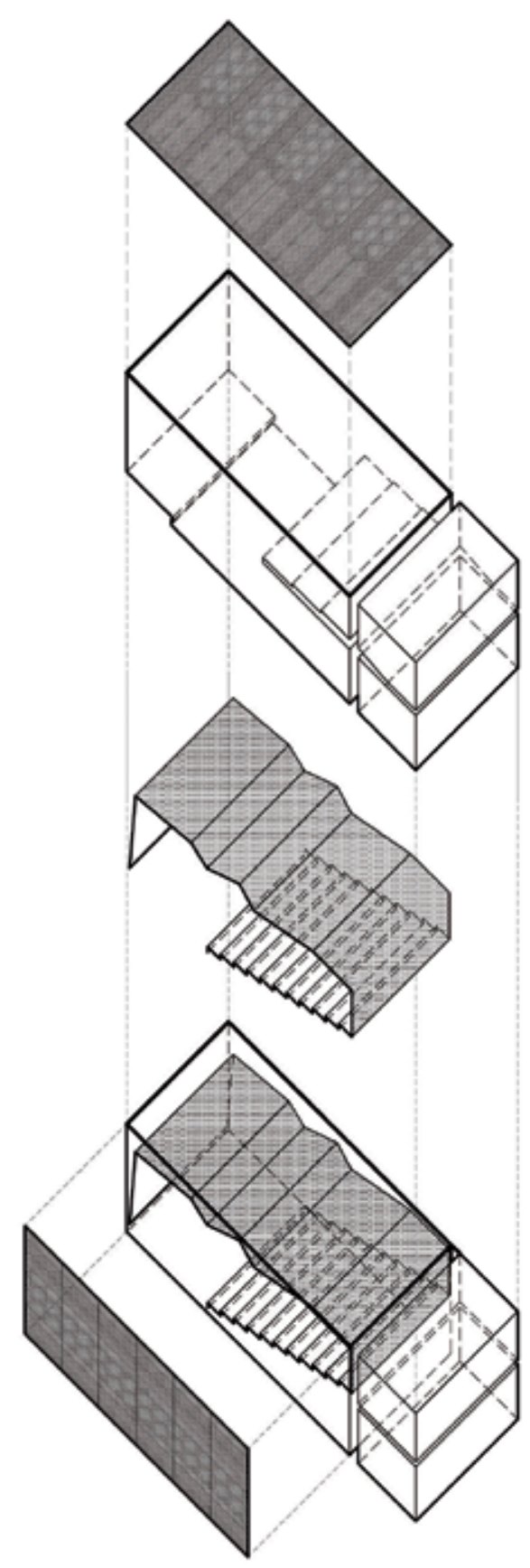

ESTRATEGIAS DE DISEÑO $\wedge$

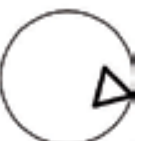


6. Detalle constructivo.

$(6$

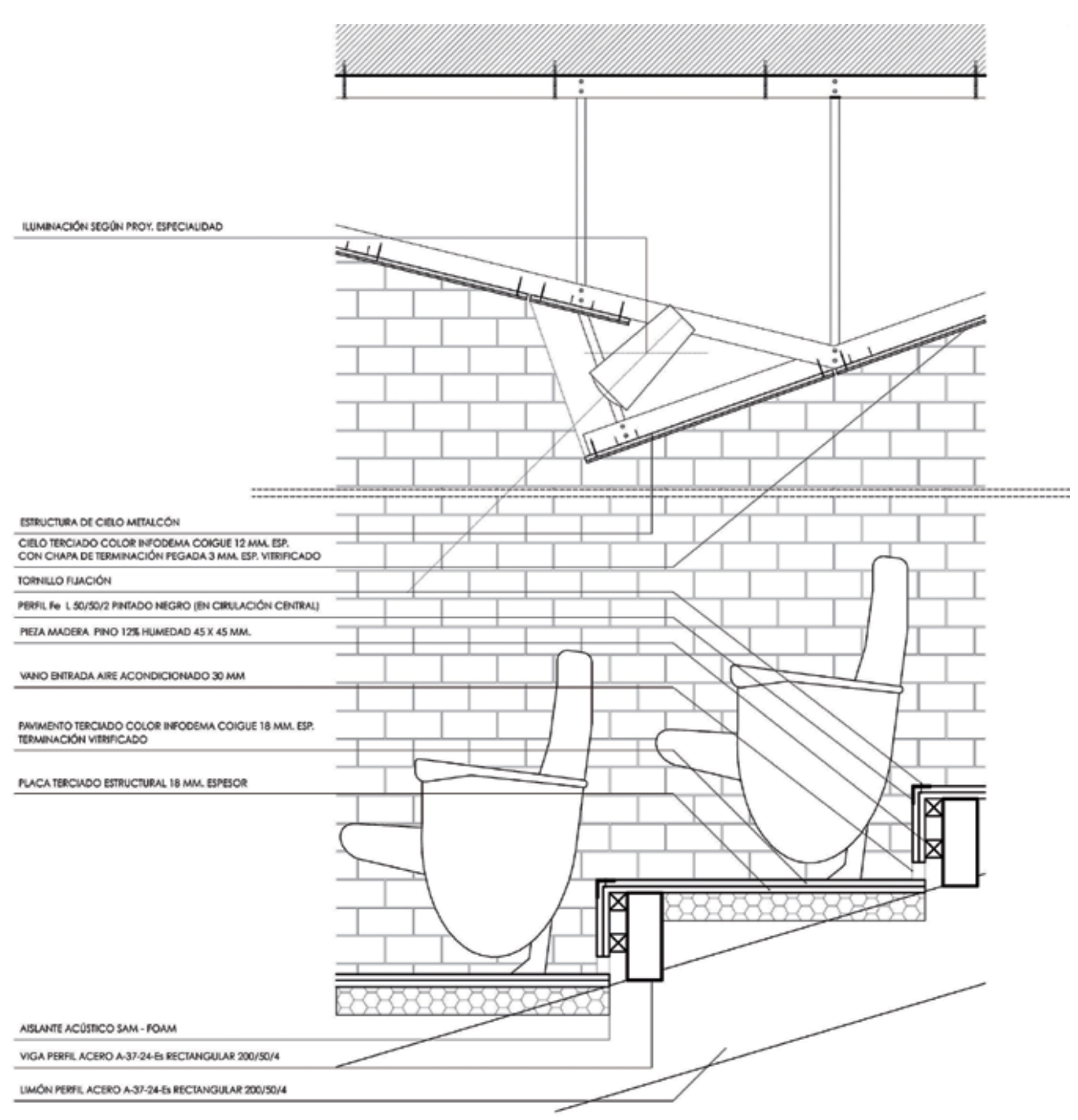

$\wedge$ DETALLES

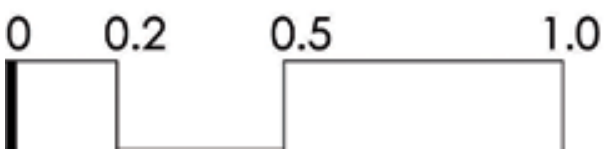


7. Plantas.

8. Cortes.

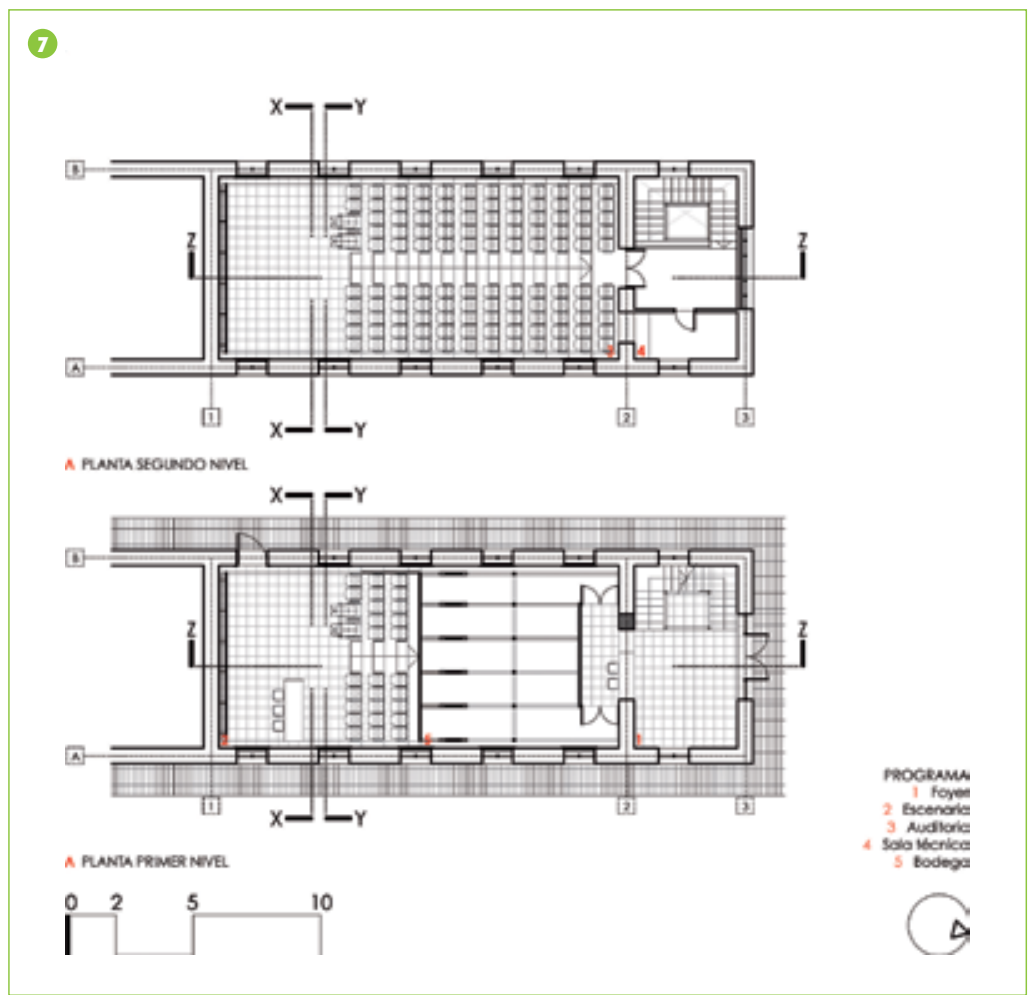

8

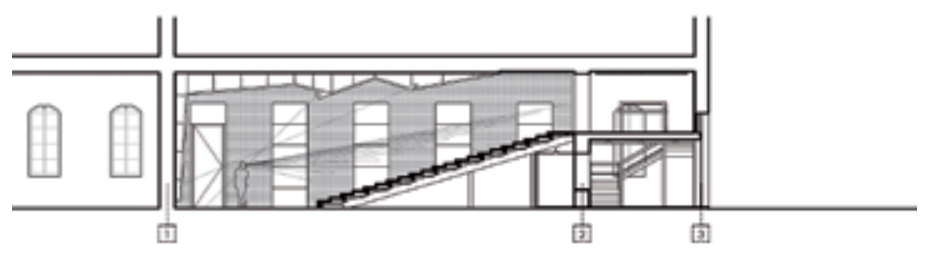

$\mathrm{Asecod} 2 z$

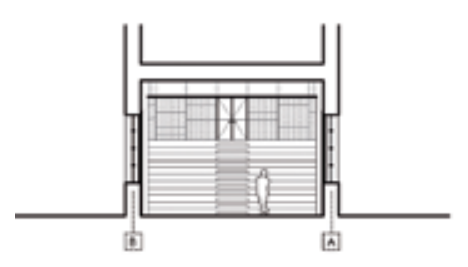

A secoón YY

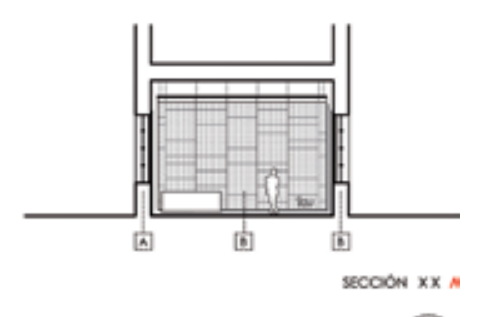

$\prod^{0} 2 \quad 5$
9. Hall del Auditorium.

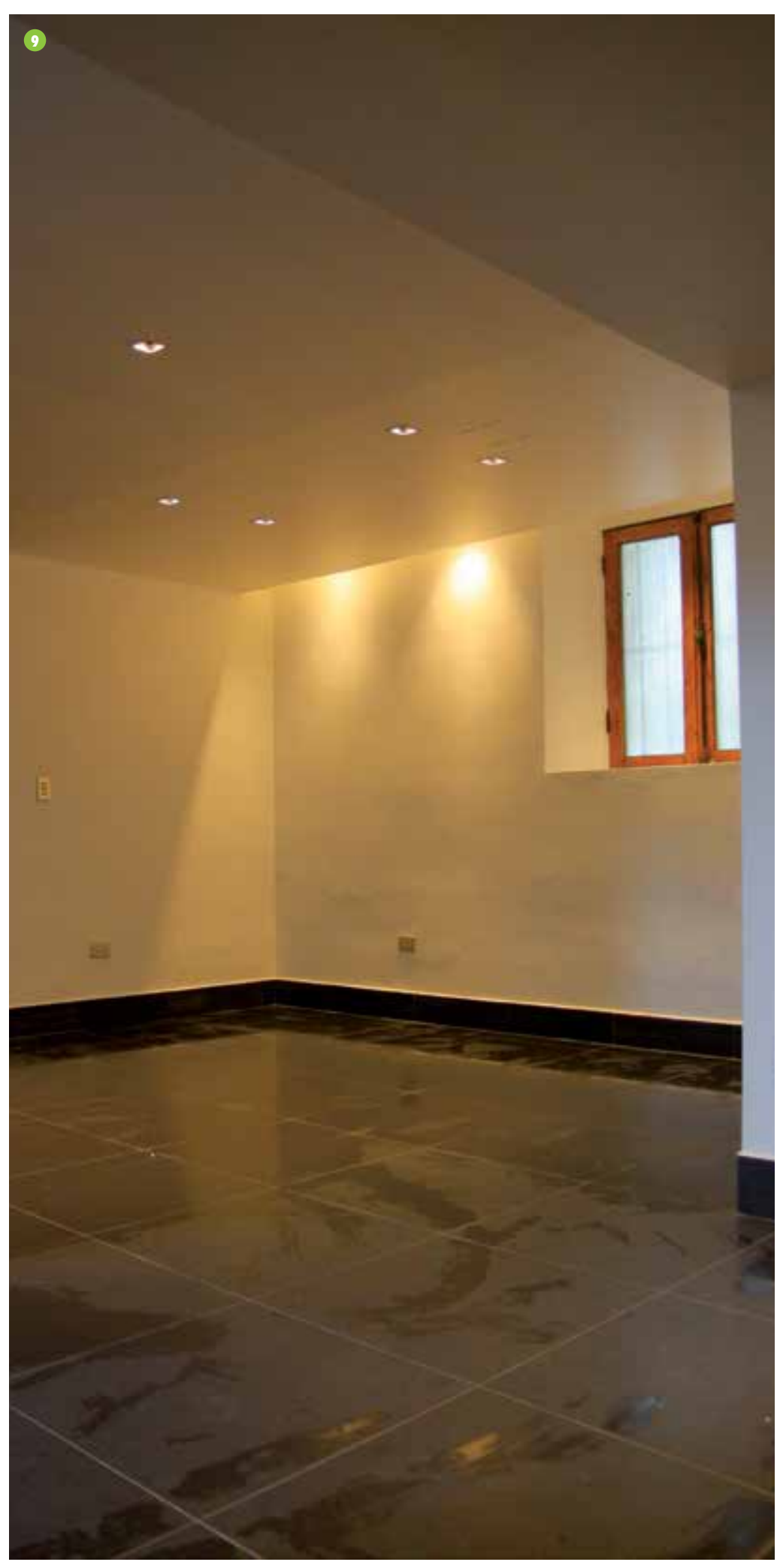


10. Escala de acceso al Auditorium.

11. Detalle del cielo del Auditorium.
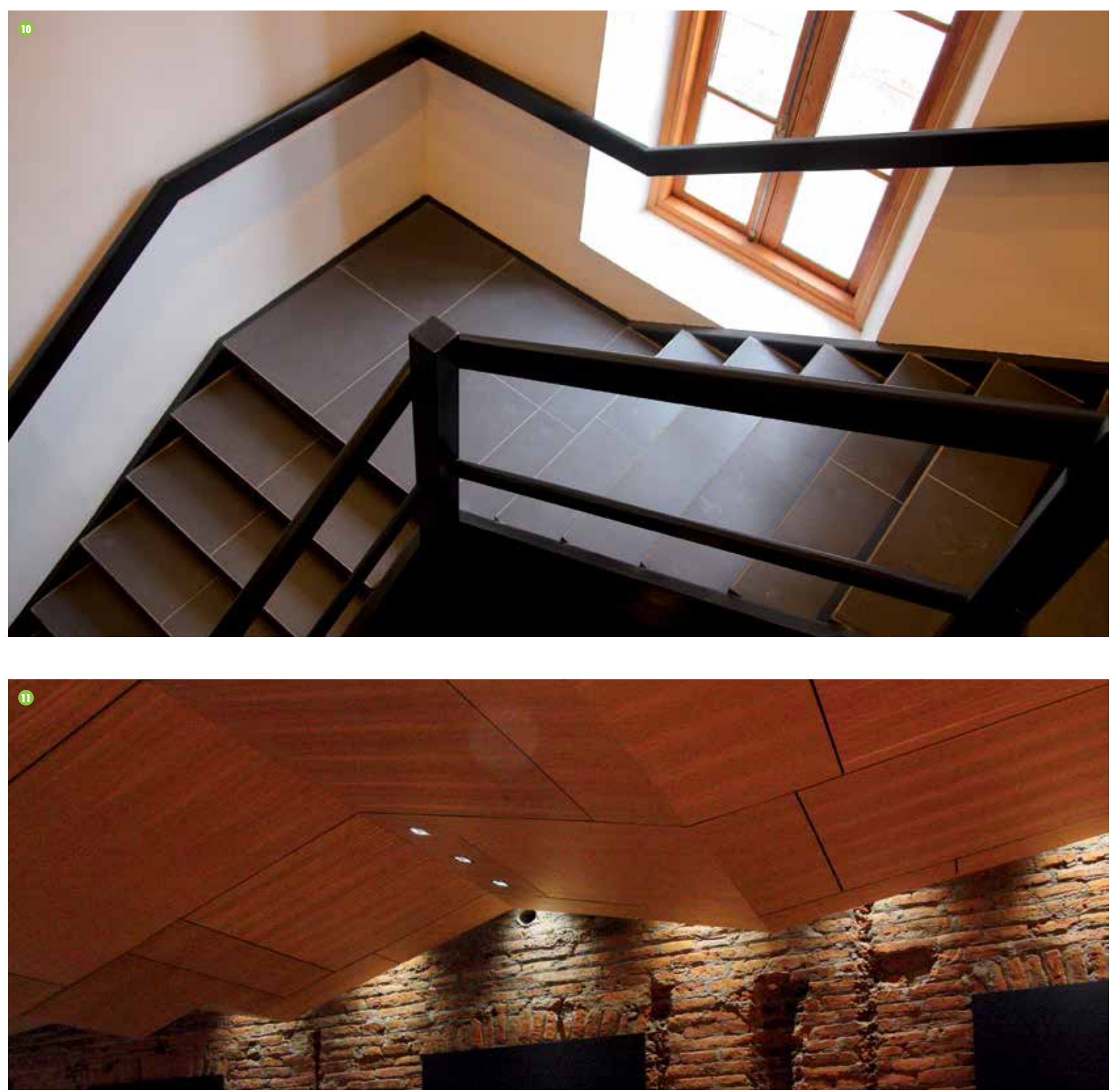


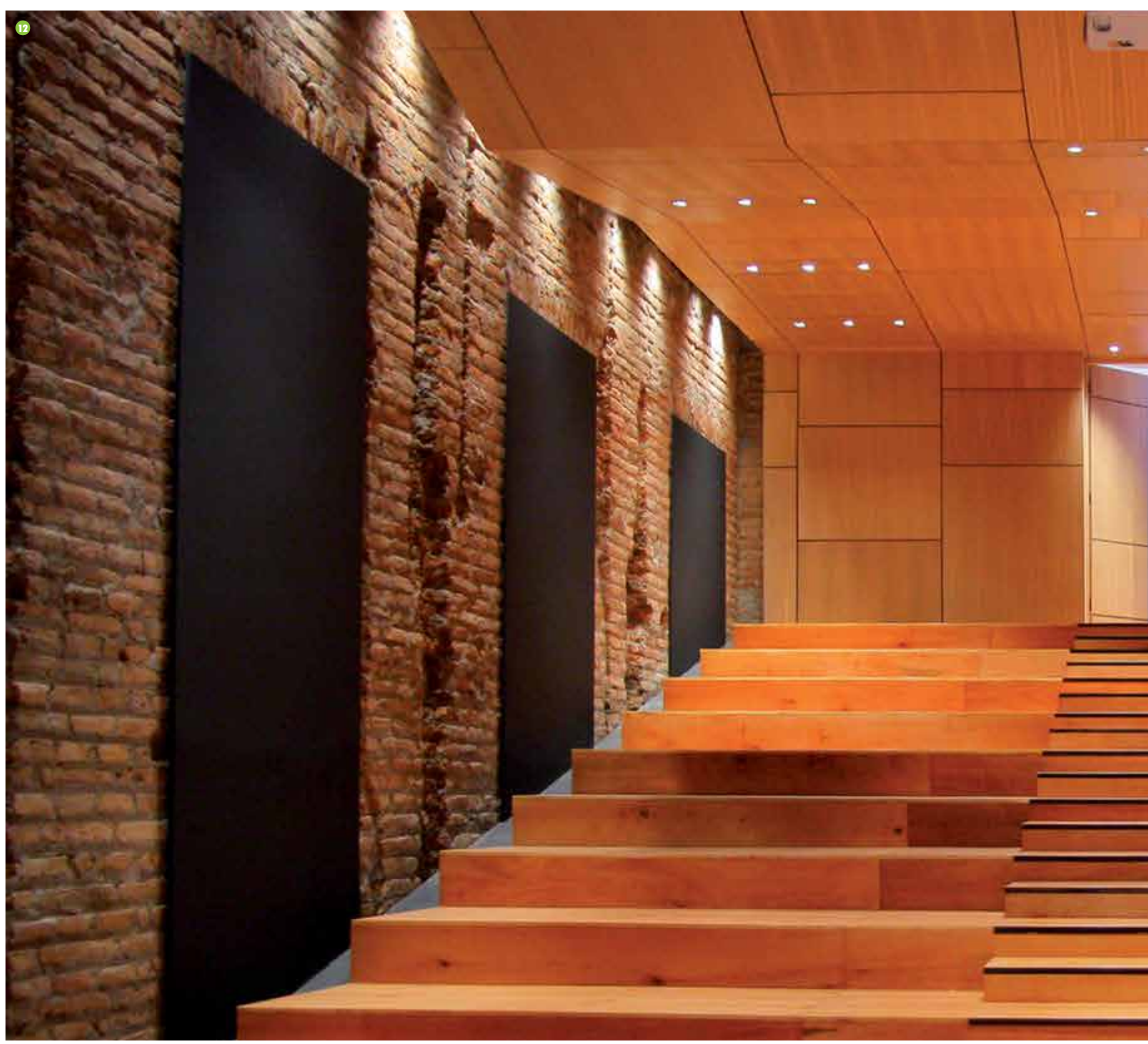


